\title{
Computational Analysis of Queues with Catastrophes in a Multiphase Random Environment
}

\author{
G. Arul Freeda Vinodhini ${ }^{1}$ and V. Vidhya ${ }^{2}$ \\ ${ }^{1}$ Bharathiar University and SSE, Saveetha University, Chennai, India \\ ${ }^{2}$ SSE, Saveetha University, Chennai, India \\ Correspondence should be addressed to G. Arul Freeda Vinodhini; tgdavid@rediffmail.com
}

Received 2 March 2016; Accepted 27 June 2016

Academic Editor: Anna Vila

Copyright (c) 2016 G. A. F. Vinodhini and V. Vidhya. This is an open access article distributed under the Creative Commons Attribution License, which permits unrestricted use, distribution, and reproduction in any medium, provided the original work is properly cited.

\begin{abstract}
A dynamically changing road traffic model which occasionally suffers a disaster (traffic collision, wrecked vehicles, conflicting weather conditions, spilled cargo, and hazardous matter) resulting in loss of all the vehicles (diverted to other routes) has been discussed. Once the system gets repaired, it undergoes $n-1$ trial phases and finally reaches its full capacity phase $n$. We have obtained the explicit steady state probabilities of the system via Matrix Geometric Method. Probability Generating Function is used to evaluate the time spent by the system in each phase. Various performance measures like mean traffic, average waiting time of vehicles, and fraction of vehicles lost are calculated. Some special cases have also been discussed.
\end{abstract}

\section{Introduction}

Qualitative behavior of Markovian Arrival Process (MAP) and its mathematical descriptions are discussed in Gross [1] and Neuts [2]. Queueing models with disaster are seen in call center applications, computer networks, or telecommunication applications which depend upon satellites [3-10], manufacturing process, and all means of transportations. In this paper, we have considered a system which undergoes a random disastrous failure, resulting in all vehicles to be cleared out of the system (directed to other roadways) and thus lost. The system then undergoes a repair phase 0 having duration which follows exponential distribution. Being repaired, the system will move to its full capacity after succeeding all its trial phases. System may fail at any of these trial phases with probability $1-q_{i}$ and go to repair phase 0 or succeed with probability $q_{i}$ and move to its next phase. Once it has succeeded all its trial phases and reached its full capacity, it may continue in that phase until a disaster occurs and goes to phase 0. In 1973, Yechiali [9] has introduced the generalization of the abovementioned model but concluded that analytical solution of such a model is not possible. Paz and Yechiali [4] have considered a similar type of system with $n$ phases, where the system after repair is allowed to go to any one of its other phases and stay there itself until a failure occurs. The transition between the operating phases is not allowed. But in many practical situations whenever failure occurs the system is repaired and it undergoes many trial phases to reach its full capacity. Taking this situation in mind, the present model has been formulated. Sophia and Praba [5] introduced one trial phase with many operating phases. Here, we take repair phase $0, n-1$ trial phases, and only one full capacity phase $n$ which is more relevant to road traffic and metro models. Transient solution of the model proposed by Paz and Yechiali [4] was solved by Udayabaskaran and Dora Pravina [7]. $M / G / 1$ queue in multiphase random environment with disasters was analyzed by Jiang et al. [6]. It has been well studied that it is a new approach of dynamically changing road traffic problems and metro projects.

The rest of the paper has been organized as follows. The mathematical model of two-dimensional random processes is formulated in Section 2. Section 3 gives the probabilities of the system in steady state which is derived explicitly using Matrix Geometric Method. The time spent by the system in each phase is calculated in Section 4 by using Probability Generating Function method. Various other performance 
measures are described in Section 5. In the mathematical model discussed above, the vehicles are allowed to arrive when the system is down. A special case when the vehicles are not allowed when the system is in repair phase is considered and the validity of the model is discussed in Section 6. The results obtained by both the methods are proved to be the same by using numerical examples in Section 7. Section 8 gives the conclusion and finally the references are listed.

\section{The Mathematical Model}

2.1. Model. Consider a $M / M / 1$ type queue with repair phase $0, n-1$ trial phases, and only one full capacity phase $n$ in an underlying random environment. This special random environment is an $n+1$-dimensional continuous time Markov chain, with phases $i=0,1,2, \ldots,(n-1), n$ governed by the probability transition matrix given below. A similar type of continuous time Markov chain with $n$ phases is discussed in $[4,5,7]$ :

$$
Q=\left(\begin{array}{cccccc}
0 & 1 & 0 & 0 & & 0 \\
1-q_{1} & 0 & q_{1} & 0 & & 0 \\
1-q_{2} & 0 & 0 & q_{2} & \cdots & 0 \\
\vdots & \vdots & \vdots & \vdots & \ddots & \vdots \\
1-q_{n-1} & 0 & 0 & 0 & \cdots & q_{n-1} \\
1 & 0 & 0 & 0 & & 0
\end{array}\right)
$$

In the repair phase $i=0$, the arrival of the customers is continuous at the rate of $\lambda_{0}$ but service is not rendered. When in phase $i \geq 1$, the system behaves as a $M\left(\lambda_{i}\right) / M\left(\mu_{i}\right) / 1$ queue, with arrival rate $\left(\lambda_{i}\right)$ and service rate $\left(\mu_{i}\right)$ which follows Poisson distribution. The time spent by the system in phase $i$ has mean $1 / \eta_{i}$ which is exponentially distributed. The system moves to trial phase 1 once the repair is completed. After residing in phase 1 for $1 / \eta_{1}$ time, it moves to phase 2 with probability $q_{1}$. In case of any disaster in phase 1 , it goes to phase 0 with probability $1-q_{1}$. Thus, it travels from phase 1 through phases $2,3, \ldots,(n-1)$ provided no disaster takes place in any of these phases and finally reaches phase $n$. In case a disaster happens in any of these phases, the system goes to phase 0 and restarts its switch over process. The system resides in phase $n$ until a disaster happens and sends it to phase 0 . Such disasters are studied in $[3,8]$.

2.2. Balance Equations. The stochastic process $\{X(t), Y(t)\}$ under consideration is two-dimensional and describes the system at any time $t$ as follows. System state is denoted by $X(t)$ [0: repair phase; $1,2,3, \ldots,(n-1)$ : trial phases; $n$ :full capacity phase] and $Y(t)$ denotes the number of customers in the system $[0,1,2,3, \ldots]$. The bivariate process $\{X(t), Y(t) ; t \geq 0\}$ is a Markov chain with state space $S=\{0,1,2, \ldots, n\} \times Z_{+}$, where $Z_{+}=\{0,1,2, \ldots\}$.

Let $P_{i, j}=\lim _{t \rightarrow \infty} P[X(t)=i, Y(t)=j]$ represent the joint steady state probability of the system in server state $i$ and number of customers $j$. Then, the differential difference equation of the system is given by

$$
\begin{gathered}
P_{00}^{\prime}(t)=-\left(\lambda_{0}+\eta_{0}\right) P_{00}(t)+\sum_{j=1}^{n} \eta_{j}\left(1-q_{j}\right) \sum_{i=0}^{\infty} P_{j, i}(t) \\
\text { when } i=0 ; m=0, \\
P_{0 m}^{\prime}(t)=-\left(\lambda_{0}+\eta_{0}\right) P_{0 m}(t)+\lambda_{0} P_{0, m-1}(t)
\end{gathered}
$$

when $i=0 ; m \geq 1$,

$$
\begin{aligned}
P_{10}^{\prime}(t)= & -\left(\lambda_{1}+\eta_{1}\right) P_{10}(t)+\eta_{0} P_{00}(t)+\mu_{1} P_{11}(t) \\
\text { when } i=1 ; m=0 & \\
P_{1 m}^{\prime}(t)= & -\left(\lambda_{1}+\mu_{1}+\eta_{1}\right) P_{1 m}(t)+\lambda_{1} P_{1, m-1}(t) \\
& +\eta_{0} P_{0 m}(t)+\mu_{1} P_{1, m+1}(t)
\end{aligned}
$$

when $i=1 ; m \geq 1$,

$$
\begin{aligned}
& P_{i 0}^{\prime}(t)=-\left(\lambda_{i}+\eta_{i}\right) P_{i 0}(t)+\eta_{i-1} q_{i-1} P_{i-1,0}(t) \\
&+\mu_{i} P_{i 1}(t) \quad \text { when } 1<i \leq n ; m=0, \\
& P_{i m}^{\prime}(t)=-\left(\lambda_{i}+\mu_{i}+\eta_{i}\right) P_{i m}(t)+\lambda_{i} P_{i, m-1}(t) \\
&+\eta_{i-1} q_{i-1} P_{i-1, m}(t)+\mu_{i} P_{i, m+1}(t) \\
& \text { when } i=1 ; m \geq 1 .
\end{aligned}
$$

\section{Matrix Geometric Method}

3.1. Level Dependent QBD Process. The steady state solution of the abovementioned model can be solved by using Neuts [2] approach. Matrix Geometric Method is used to calculate steady state probabilities of Markovian arrivals [11]. The level dependent QBD bivariate process $\{X(t), Y(t)\}$ with infinitesimal generator $Q$ is given as follows:

$$
Q=\left[\begin{array}{cccccc}
B+A_{2}+A_{1} & A_{0} & O & O & O & \cdots \\
B+A_{2} & A_{1} & A_{0} & O & O & \cdots \\
B & A_{2} & A_{1} & A_{0} & O & \cdots \\
B & O & A_{2} & A_{1} & A_{0} & \cdots \\
\vdots & \vdots & \vdots & \vdots & \vdots & \ddots
\end{array}\right],
$$

where $O$ is the zero matrices with appropriate dimension and $A_{i}$ and $B$ are square matrices of order $N+1$ given as follows:

$$
B=\left[\begin{array}{cccccc}
0 & 0 & 0 & 0 & \cdots & 0 \\
\eta_{1}\left(1-q_{1}\right) & 0 & 0 & 0 & \cdots & 0 \\
\eta_{2}\left(1-q_{2}\right) & 0 & 0 & 0 & \cdots & 0 \\
\eta_{3}\left(1-q_{3}\right) & 0 & 0 & 0 & \cdots & 0 \\
\vdots & \vdots & \vdots & \vdots & \ddots & 0 \\
\eta_{n-1}\left(1-q_{n-1}\right) & 0 & 0 & 0 & \cdots & 0 \\
\eta_{n} & 0 & 0 & 0 & \cdots & 0
\end{array}\right],
$$




$$
\begin{aligned}
A_{0} & =\operatorname{diag}\left(\lambda_{0}, \lambda_{1}, \lambda_{2}, \ldots, \lambda_{n}\right), \\
A_{1} & =\left[\begin{array}{lllllllll}
a & b & & & & & & & \\
\mu & c_{1} & d_{1} & & & & & & \\
& \mu & c_{2} & d_{2} & & & & & \\
& & \mu & c_{3} & d_{3} & & & & \\
& & & \mu & c_{4} & d_{4} & & & \\
& & & & \mu & c_{5} & & & \\
& & & & & & \ddots & & \\
& & & & & & & & \\
& & & & & & & c_{n-1} & d_{n-1} \\
& & & & & & & \mu & c_{n}
\end{array}\right],
\end{aligned}
$$

where $a=-\lambda_{0}-\eta_{0}, b=\eta_{0}, c_{n}=-\lambda_{n}-\mu_{n}-\eta_{n}$, and $d_{n}=\eta_{n} q_{n}$ :

$$
A_{2}=\operatorname{diag}\left(0, \mu_{1}, \mu_{2}, \ldots, \mu_{n}\right) \text {. }
$$

Lemma 1. The necessary and sufficient condition for the process $X(t)$ to be positive recurrent is $\lambda_{n} / \mu_{n}<1$.

The following system of equations has the stationary distribution solution $\pi$ :

$$
\begin{aligned}
\pi Q & =O, \\
\pi e & =1 .
\end{aligned}
$$

Here, vector e represents a column vector with entries as ones and vector $O$ represents a row vector with an appropriate dimension whose entries are zeros.

Let $\pi=\pi_{0}, \pi_{1}, \pi_{2}, \pi_{3}, \ldots$ with

$$
\begin{aligned}
& \pi_{0}=\left[\begin{array}{lllll}
P_{00} & P_{10} & P_{20} & \cdots & P_{n 0}
\end{array}\right], \\
& \pi_{k}=\left[\begin{array}{lllll}
P_{0 k} & P_{1 k} & P_{2 k} & \cdots & P_{n k}
\end{array}\right],
\end{aligned}
$$

where $k=1,2, \ldots, \infty$. Then,

$$
\begin{aligned}
\pi_{m} & =\pi_{0} R^{m} \quad m \geq 1, \\
\pi_{0}\left[(I-R)^{-1} B+A_{1}+A_{2}+R A_{2}\right] & =0 .
\end{aligned}
$$

Implying that

$$
\sum_{m=0}^{\infty} \pi_{m} e=\pi_{0}\left(\sum_{m=0}^{\infty} R^{m}\right) e=\pi_{0}\left((I-R)^{-1}\right) e=1,
$$

here, $R$ is a square matrix of order $n+1$ and is the solution of the following equation:

$$
R^{2} A_{2}+R A_{1}+A_{0}=0 .
$$

3.2. Evaluation of R Matrix. As the matrices $A_{2}, A_{1}$, and $A_{0}$ have upper diagonal form, it follows from (11) that matrix $R$ will also have the upper diagonal structure as follows:

$$
R=\left[\begin{array}{ccccc}
r_{00} & r_{01} & r_{02} & & r_{0 n} \\
0 & r_{11} & r_{12} & \cdots & r_{1 n} \\
0 & 0 & r_{22} & & r_{2 n} \\
& \vdots & & \ddots & \vdots \\
0 & 0 & 0 & \cdots & r_{n n}
\end{array}\right] .
$$

Using (11) and (12), the nonzero entries of $R$ are given by

$$
\begin{aligned}
r_{00} & =\frac{\lambda_{0}}{\lambda_{0}+\eta_{0}}, \\
r_{i i}= & \frac{\left(\lambda_{i}+\mu_{i}+\eta_{i}\right)-\sqrt{\left(\lambda_{i}+\mu_{i}+\eta_{i}\right)^{2}-4 \mu_{i} \lambda_{i}}}{2 \mu_{i}} \\
r_{01}= & \text { for } i=1,2,3, \ldots, n, \\
r_{i, i+1}= & \frac{\eta_{0} r_{00}}{\left.\left(\lambda_{1}+\mu_{1}+\eta_{1}\right)-\mu_{1} r_{00}-\mu_{1} r_{11}+\mu_{i+1}+\eta_{i+1}\right)-\mu_{i+1}\left(r_{i i}+r_{i+1, i+1}\right)}, \\
r_{i, i+j}= & \frac{\eta_{i+j-1} q_{i+j-1} r_{i, i+j-1}+\mu_{i+j}\left\{\sum_{k=i+1}^{i+j-1} r_{i k} r_{k, i+j}\right\}}{\left(\lambda_{i+j}+\mu_{i+j}+\eta_{i+j}\right)-\mu_{i+j}\left(r_{i i}+r_{i+j, i+j}\right)} \\
\quad j=2,3,4, \ldots, n, i=0,1,2, \ldots, n-j . & i=1,2,3, n-1,
\end{aligned}
$$

3.3. Calculation of $\pi_{i}$. Using matrix $R$ in (9) and normalizing the obtained vector $g_{0}$ with $g_{0}\left((I-R)^{-1}\right) e=\alpha$, we get $\pi_{0}$. Using (8), the remaining $\pi_{i} i \geq 1$ can be calculated. Thus, the entire system is defined.

\section{Probability Generating Function}

Under steady state conditions, (2) becomes

$$
\begin{aligned}
\left(\lambda_{0}+\eta_{0}\right) P_{00} & =\sum_{j=1}^{n} \eta_{j}\left(1-q_{j}\right) \sum_{i=0}^{\infty} P_{j, i} \\
& =\sum_{j=1}^{n} \eta_{j}\left(1-q_{j}\right) P_{j} .
\end{aligned}
$$

$$
\begin{aligned}
\text { when } i=0 ; m=0, & \\
\left(\lambda_{0}+\eta_{0}\right) P_{0 m}= & \lambda_{0} P_{0, m-1} \quad \text { when } i=0 ; m \geq 1, \\
\left(\lambda_{1}+\eta_{1}\right) P_{10}=\eta_{0} P_{00}(t)+\mu_{1} P_{11} & \text { when } i=1 ; m=0, \\
\left(\lambda_{1}+\mu_{1}+\eta_{1}\right) P_{1 m}= & \lambda_{1} P_{1, m-1}+\eta_{0} P_{0 m}(t)+\mu_{1} P_{1, m+1} \\
\text { when } i=1 ; m \geq 1, & \text { when } 1<i \leq n ; m=0, \\
\left(\lambda_{i}+\eta_{i}\right) P_{i 0}= & \eta_{i-1} q_{i-1} P_{i-1,0}+\mu_{i} P_{i 1} \\
\left(\lambda_{i}+\mu_{i}+\eta_{i}\right) P_{i m}= & \lambda_{i} P_{i, m-1}+\eta_{i-1} q_{i-1} P_{i-1, m} \\
& +\mu_{i} P_{i, m+1}
\end{aligned}
$$


Substituting $Q$ of this model into $q_{i j}$ of Yechiali [9], the above mentioned steady state equations coincide with steady state equations of Yechiali [9].

Consider Probability Generating Functions defined as follows [see [1]]:

$$
G_{i}(z)=\sum_{m=0}^{\infty} P_{i m} z^{m} \quad \text { for } i=0,1,2, \ldots, n .
$$

Then,

$$
G_{i}(1)=\sum_{m=0}^{\infty} P_{i m}=P_{i} .
$$

Also,

$$
\sum_{i=1}^{n} G_{i}(1)=1
$$

4.1. Proposition. The probability of zero customers in phase 0 is given by

$$
P_{00}=\left(\lambda_{0}+\eta_{0}\right)^{-1}\left[\frac{1}{\eta_{0}}+\frac{1}{\eta_{1}}+\sum_{i=2}^{n} \frac{\prod_{m=1}^{i-1} q_{m}}{\eta_{i}}\right]^{-1} .
$$

Proof. Using (20) in (15), we prove that

$$
\left(\lambda_{0}+\eta_{0}-z \lambda_{0}\right) G_{0}(z)=\left(\lambda_{0}+\eta_{0}\right) P_{00}
$$

Putting $z=1$ in (24), we get $P_{0}=\left(\left(\lambda_{0}+\eta_{0}\right) / \eta_{0}\right) P_{00}$.

Using (20) in (17), we prove that

$$
\begin{gathered}
\left(\lambda_{1}+\mu_{1}+\eta_{1}-z \lambda_{1}-\frac{\mu_{1}}{z}\right) G_{1}(z) \\
=\eta_{0} G_{0}(z)-\mu_{1} P_{10}\left[\frac{1}{z}-1\right] .
\end{gathered}
$$

Putting $z=1$ in (25), we get $P_{1}=\left(\left(\lambda_{0}+\eta_{0}\right) / \eta_{1}\right) P_{00}$.

Using (20) in (19), we prove that

$$
\begin{aligned}
& \left(\lambda_{i}+\mu_{i}+\eta_{i}-z \lambda_{i}-\frac{\mu_{i}}{z}\right) G_{i}(z) \\
& =\eta_{i-1} q_{i-1} G_{i-1}(z)-\mu_{i} P_{i 0}\left[\frac{1}{z}-1\right] \\
& \quad i=2,3, \ldots, n .
\end{aligned}
$$

Putting $z=1$ in (26), we get

$$
P_{i .}=\frac{\eta_{i-1} q_{i-1}}{\eta_{i}} P_{(i-1) \cdot}, \quad i=2,3, \ldots, n .
$$

Using recursively the abovementioned equations, we prove that

$$
P_{i .}=\frac{\lambda_{0}+\eta_{0}}{\eta_{i}} \prod_{m=1}^{i-1} q_{m} P_{00} .
$$

Using these results in (22), we prove that

$$
P_{00}=\left(\lambda_{0}+\eta_{0}\right)^{-1}\left[\frac{1}{\eta_{0}}+\frac{1}{\eta_{1}}+\sum_{i=2}^{n}\left(\frac{\prod_{m=1}^{i-1} q_{m}}{\eta_{i}}\right)\right]^{-1} .
$$

4.2. To Find the Probability That the System Resides in Phase 0 . With the direct usage of (15) recursively, we get

$$
P_{0 m}=\left(\frac{\lambda_{0}}{\lambda_{0}+\eta_{0}}\right)^{m} P_{00} \quad m \geq 1 .
$$

Hence, $P_{0 i}$ is found for $i=1,2, \ldots, \infty$.

Thus, the proportion of time in which the system resides in phase 0 is $=P_{0}=\left(\left(\lambda_{0}+\eta_{0}\right) / \eta_{0}\right) P_{00}$.

These probabilities coincide with Yechiali [9].

4.3. To Find the Probability That the System Resides in Phase $i$. From (24), we get $G_{0}(z)=\left(\left(\lambda_{0}+\eta_{0}\right) /\left(\lambda_{0}+\eta_{0}-z \lambda_{0}\right)\right) P_{00}$.

Rearranging (25) and (26), we get

$$
\begin{aligned}
& G_{1}(z)\left[-\lambda_{1} z^{2}+\left(\lambda_{1}+\mu_{1}+\eta_{1}\right) z-\mu_{1}\right] \\
& =(z-1) \mu_{1} P_{10}+\eta_{0} z G_{0}(z) \\
& G_{i}(z)\left[-\lambda_{i} z^{2}+\left(\lambda_{i}+\mu_{i}+\eta_{i}\right) z-\mu_{i}\right] \\
& \quad=(z-1) \mu_{i} P_{i 0}+\eta_{i-1} q_{i-1} z G_{i-1}(z) .
\end{aligned}
$$

Define

$$
\begin{aligned}
& \phi_{0}(z)=\lambda_{0}(1-z)+\eta_{0} \\
& \phi_{i}(z)=-\lambda_{i} z^{2}+\left(\lambda_{i}+\mu_{i}+\eta_{i}\right) z-\mu_{i} \quad i \geq 1 .
\end{aligned}
$$

Each quadratic polynomial $\phi_{i}(z) i \geq 1$ has two roots that are real. The only root of $\phi_{i}(z)$ which lies in the interval $(0,1)$ is denoted by $z_{i}$. This result holds because

$$
\begin{aligned}
\phi_{i}(0) & =-\mu_{i}<0, \\
\phi_{i}(1) & =\eta_{i}>0, \\
\phi_{i}( \pm \infty) & <0 .
\end{aligned}
$$

The root $z_{i} \in(0,1)$ is given by

$$
z_{i}=\frac{\left(\lambda_{i}+\mu_{i}+\eta_{i}\right)-\sqrt{\left(\lambda_{i}+\mu_{i}+\eta_{i}\right)^{2}-4 \lambda_{i} \mu_{i}}}{2 \lambda_{i}} .
$$

Here, $z_{i}$ represents the LST (Laplace Stieltjes Transform), evaluated at point $\eta_{i}$, of the busy period in a $M / M / 1$ queue with arrival rate $\lambda_{i}$ and service rate $\mu_{i}$.

Substituting $z=z_{1}$ into (31), we can easily prove that

$$
P_{10}=\frac{\eta_{0}}{\mu_{1}}\left(\lambda_{0}+\eta_{0}\right) \frac{z_{1}}{\left(1-z_{1}\right)^{2} \lambda_{0}+\left(1-z_{1}\right) \eta_{0}} P_{00} \text {. }
$$

Thus, each PGF $G_{i}(z)$ is completely determined by the abovementioned equations. Any probability $P_{i m}$ can be calculated by differentiating $G_{i}(z)$ and substituting $z=0$.

The probability that the system resides in phase 1 is

$$
P_{1 \cdot}=\left(\frac{\eta_{0}}{\eta_{1}}\right) P_{0} \text {. }
$$

The probability that the system resides in phase $i$ is

$$
P_{i .}=\left(\frac{\eta_{i-1} q_{i-1}}{\eta_{i}}\right) P_{(i-1) \cdot}, \quad 2 \leq i \leq n .
$$




\section{Performance Measures}

As $G_{i}(z)=\sum_{m=0}^{\infty} P_{\text {im }} z^{m}, G_{i}^{\prime}(z)=\sum_{m=1}^{\infty} m P_{\text {im }} z^{m-1}$.

The number of customers in $i$ th phase $=L_{i}=G_{i}^{\prime}(1)=$ $\sum_{m=1}^{\infty} m P_{i m}$.

Thus,

$$
\begin{array}{rl}
L_{0} & =\frac{\lambda_{0}}{\lambda_{0}+\eta_{0}} P_{00}, \\
L_{1} & =\frac{\eta_{0}}{\eta_{1}} L_{0}+\frac{\eta_{0}\left[\lambda_{1}-\mu_{1}\right]}{\eta_{1}^{2}} P_{0 .}+\frac{\mu_{1}}{\eta_{1}} P_{10}, \\
L_{i}= & \frac{\eta_{i-1} q_{i-1}}{\eta_{i}} L_{i-1}+\frac{\eta_{i-1} q_{i-1}\left[\lambda_{i}-\mu_{i}\right]}{\eta_{i}^{2}} P_{i .}+\frac{\mu_{i}}{\eta_{i}} P_{i 0} \\
i & i=2,3,4, \ldots, n .
\end{array}
$$

The number of customers in the system at any time is = $\sum_{i=0}^{n} L_{i}$.

Let $C$ be the number of customers cleared from the system per unit time. Then, $E(C)=\sum_{i=1}^{n} \eta_{i} L_{i}$.

The fraction of customers receiving full service is therefore $1-E(C) / \lambda$.

\section{Special Cases}

6.1. Validation of the Model [No Trial Phase]. When there are no trials in between (ie) after repair phase 0 , the system directly moves to the full capacity phase; the general model introduced here coincides with Yechiali [10] with $\xi=0$. Assuming $n=1$, the system goes to full capacity phase $q_{1}=1$ directly. Also all the results obtained become

$$
\begin{aligned}
& P_{00}=\frac{1}{\left(\lambda_{0}+\eta_{0}\right)} \frac{\eta_{0} \eta_{1}}{\left(\eta_{1}+\eta_{0}\right)}, \\
& P_{0 .}=\frac{\eta_{1}}{\eta_{1}+\eta_{0}}, \\
& P_{1 .}=\frac{\eta_{0}}{\eta_{1}+\eta_{0}}, \\
& P_{10}=\frac{\eta_{0}^{2}}{\mu_{1}} \frac{z_{1}}{\left[\left(1-z_{1}\right)^{2} \lambda_{0}+\left(1-z_{1}\right) \eta_{0}\right]} P_{0 .},
\end{aligned}
$$

where $z_{1}$ is the root of $\phi_{1}(z)$ as discussed earlier.

6.2. Arrival Stops When System Is Down. When customers are not allowed during repair phase 0 , we have

$$
\begin{aligned}
\lambda_{0} & =0, \\
L_{0} & =0, \\
P_{0}= & P_{00}, \\
P_{10} & =\frac{\eta_{0}}{\mu_{1}} \frac{z_{1}}{\left(1-z_{1}\right)} P_{00}, \\
L_{1} & =\left[\frac{\eta_{0}\left[\lambda_{1}-\mu_{1}\right]}{\eta_{1}{ }^{2}}+\frac{\mu_{1}}{\eta_{1}} \frac{\eta_{0}}{\mu_{1}} \frac{z_{1}}{\left(1-z_{1}\right)}\right] P_{00} .
\end{aligned}
$$

\section{Numerical Example}

Consider a traffic model which on completion possesses one repair phase 0 , two trial phases 1,2 , and one full capacity phase 3 with the following data.

Case 1. Consider

$$
\begin{aligned}
& \lambda_{0}=2 \text {, } \\
& \lambda_{1}=2 \text {, } \\
& \lambda_{2}=2 \text {, } \\
& \lambda_{3}=2 \text {, } \\
& \mu_{0}=0 \text {, } \\
& \mu_{1}=5 \text {, } \\
& \mu_{2}=7 \text {, } \\
& \mu_{3}=9 \text {, } \\
& \eta_{0}=2 \text {, } \\
& \eta_{1}=4 \text {, } \\
& \eta_{2}=4 \text {, } \\
& \eta_{3}=4 \text {, } \\
& Q=\left(\begin{array}{cccc}
0 & 1 & 0 & 0 \\
0.75 & 0 & 0.25 & 0 \\
0.5 & 0 & 0 & 0.5 \\
1 & 0 & 0 & 0
\end{array}\right)
\end{aligned}
$$

Case 2 (sensitivity when $\lambda_{0}=0$ ).

$$
\begin{aligned}
& \lambda_{0}=0, \\
& \lambda_{1}=2, \\
& \lambda_{2}=2, \\
& \lambda_{3}=2, \\
& \mu_{0}=0, \\
& \mu_{1}=5, \\
& \mu_{2}=7, \\
& \mu_{3}=9, \\
& \eta_{0}=2, \\
& \eta_{1}=4, \\
& \eta_{2}=4, \\
& \eta_{3}=4,
\end{aligned}
$$


TABLE 1: Steady state probabilities.

\begin{tabular}{lllllllrr}
\hline & \multicolumn{3}{c}{ Case 1 } & \multicolumn{3}{c}{ Case 2 [sensitivity analysis] } \\
\hline$\pi_{0}$ & 0.296 & 0.123 & 0.027 & 0.010 & 0.592 & 0.190 & 0.033 \\
$\pi_{1}$ & 0.148 & 0.078 & 0.020 & 0.009 & 0.000 & 0.068 & 0.020 & 0.012 \\
$\pi_{2}$ & 0.074 & 0.045 & 0.013 & 0.006 & 0.000 & 0.024 & 0.011 \\
$\pi_{3}$ & 0.037 & 0.024 & 0.008 & 0.004 & 0.000 & 0.009 & 0.005 \\
$\pi_{4}$ & 0.018 & 0.013 & 0.005 & 0.003 & 0.000 & 0.003 & 0.003 & 0.009 \\
$\pi_{5}$ & 0.009 & 0.007 & 0.003 & 0.002 & 0.000 & 0.001 & 0.001 \\
$\pi_{6}$ & 0.005 & 0.003 & 0.001 & 0.001 & 0.000 & 0.000 & 0.001 \\
$\pi_{7}$ & 0.002 & 0.002 & 0.001 & 0.001 & 0.000 & 0.000 & 0.000 \\
$\pi_{8}$ & 0.001 & 0.001 & 0.000 & 0.000 & 0.000 & 0.000 & 0.001 \\
$\pi_{9}$ & 0.001 & 0.000 & 0.000 & 0.000 & 0.000 & 0.000 & 0.000 \\
$\pi_{10}$ & 0.000 & 0.000 & 0.000 & 0.000 & 0.000 & 0.000 & 0.000 \\
\hline
\end{tabular}

TABLE 2: The probability that the system resides in phase $i$.

\begin{tabular}{|c|c|c|c|c|}
\hline & \multicolumn{2}{|c|}{ Case 1} & \multicolumn{2}{|c|}{ Case 2 [sensitivity analysis] } \\
\hline & Matrix Geometric Method & $\begin{array}{l}\text { Probability Generating } \\
\text { Function method }\end{array}$ & Matrix Geometric Method & $\begin{array}{l}\text { Probability Generating } \\
\text { Function method }\end{array}$ \\
\hline$\overline{P_{00}}$ & 0.295605 & 0.296296 & 0.591879 & 0.592593 \\
\hline$P_{0}$ & 0.590921 & 0.592593 & 0.591879 & 0.592593 \\
\hline$P_{1}$ & 0.29603 & 0.296296 & 0.295917 & 0.296296 \\
\hline$P_{2}$ & 0.078151 & 0.074074 & 0.073983 & 0.074074 \\
\hline$P_{3}$ & 0.037353 & 0.037037 & 0.036704 & 0.037037 \\
\hline
\end{tabular}

Note. We see that probabilities calculated by both methods remain the same for both the cases and $P_{0}=P_{00}$ for Case 2 .

$$
Q=\left(\begin{array}{cccc}
0 & 1 & 0 & 0 \\
0.75 & 0 & 0.25 & 0 \\
0.5 & 0 & 0 & 0.5 \\
1 & 0 & 0 & 0
\end{array}\right)
$$

The various steady state probabilities of both the cases are listed in Table 1 and the corresponding graph showing their comparison is given as Figure 1 .

The probability that the system resides in each phase is calculated by both Matrix Geometric Method and Probability Generating Function method and listed in Table 2. It is observed that the probabilities coincide by both the methods proving the validity of our analytical results.

\section{Conclusions}

We have modeled a dynamically changing traffic model which undergoes disaster as a $M / M / 1$ queue with repair phase 0 , trial phases $1,2,3, \ldots,(n-1)$, and full capacity phase $n$. Steady state probabilities and other performance measures are calculated by two methods: Matrix Geometric Method and Probability Generating Function method. Numerical illustrations prove that the results obtained by both methods are one and the same. Also, validity of the model and sensitivity analysis are discussed as special cases.

Since the abovementioned model is more suitable for metro trains, traffic networks, and so forth, the transient

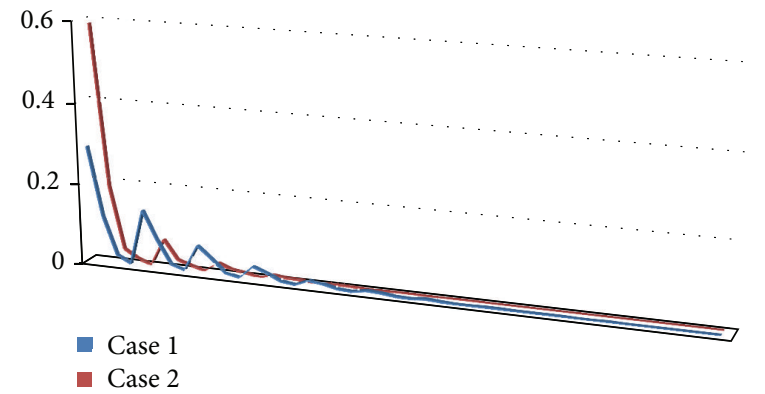

FIGURE 1: Comparison of steady state probabilities of two cases.

solution will be more suitable and hence it can be considered for future research. The abovementioned model can be treated as $M / G / 1$ queue and its steady state solution can be found.

\section{Competing Interests}

The authors declare that they have no competing interests.

\section{References}

[1] D. Gross, Fundamentals of Queuing Theory, John Wiley \& Sons, Hoboken, NJ, USA, 4th edition, 2008.

[2] M. F. Neuts, Matrix-Geometric Solutions in Stochastic Models: An Algorithmic Approach, vol. 2 of Johns Hopkins Series in the 
Mathematical Sciences, The Johns Hopkins University Press, Baltimore, Md, USA, 1981.

[3] G. A. F. Vinodhini and V. Vidhya, "Transient solution of a multiserver queue with catastrophes and impatient customers when system is down," Applied Mathematical Sciences, vol. 8, no. 92, pp. 4585-4592, 2014.

[4] N. Paz and U. Yechiali, "An $m / m / 1$ queue in random environment with disasters," Asia-Pacific Journal of Operational Research, vol. 31, no. 3, Article ID 1450016, 12 pages, 2014.

[5] S. Sophia and B. Praba, "Steady-state behavior of an M/M/1 queue in random environment subject to system failures and repairs," International Journal of Pure and Applied Mathematics, vol. 101, no. 2, pp. 267-279, 2015.

[6] T. Jiang, L. Liu, and J. Li, "Analysis of the M/G/1 queue in multi-phase random environment with disasters," Journal of Mathematical Analysis and Applications, vol. 430, no. 2, pp. 857873, 2015.

[7] S. Udayabaskaran and C. T. Dora Pravina, "Transient analysis of an $\mathrm{M} / \mathrm{M} / 1$ queue in a random environment subject to disasters," Far East Journal of Mathematical Sciences, vol. 91, no. 2, pp. 157167, 2014.

[8] V. Vidhya, "Disasters and customer impatience in bulk queueing systems," Asia Pacific Journal of Research, vol. 1, no. 8, pp. 78-83, 2013.

[9] U. Yechiali, "A queuing-type birth-and-death process defined on a continuous-time Markov chain," Operations Research, vol. 21, pp. 604-609, 1973.

[10] U. Yechiali, "Queues with system disasters and impatient customers when system is down," Queueing Systems, vol. 56, no. 3-4, pp. 195-202, 2007.

[11] B. K. Kumar, R. Rukmani, and V. Thangaraj, "Analysis of $\mathrm{MAP} / \mathrm{PH}(1), \mathrm{PH}(2) / 2$ queue with Bernoulli vacations," Journal of Applied Mathematics and Stochastic Analysis, vol. 2008, Article ID 396871, 20 pages, 2008. 


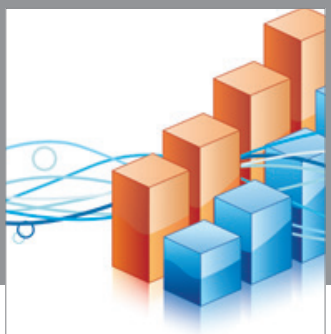

Advances in

Operations Research

vatem alat4

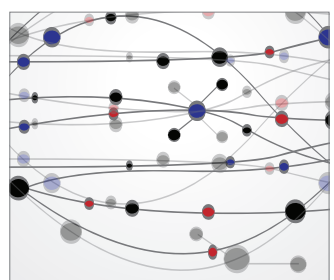

\section{The Scientific} World Journal
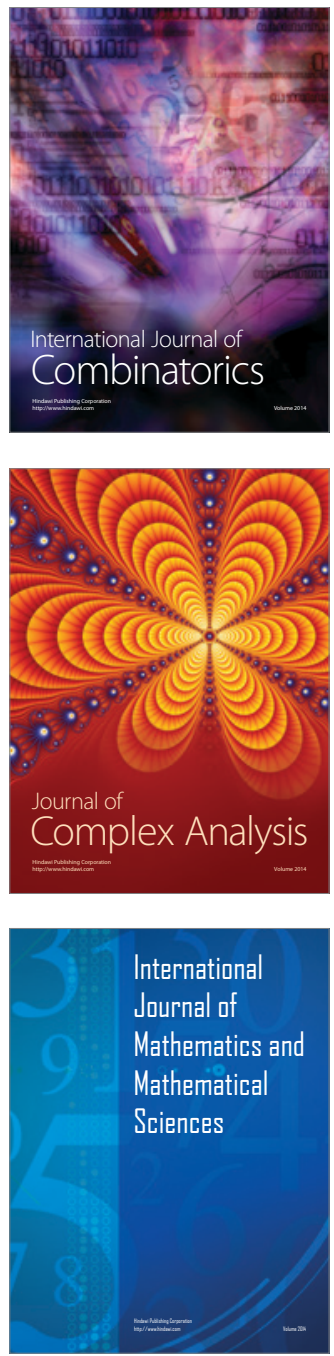
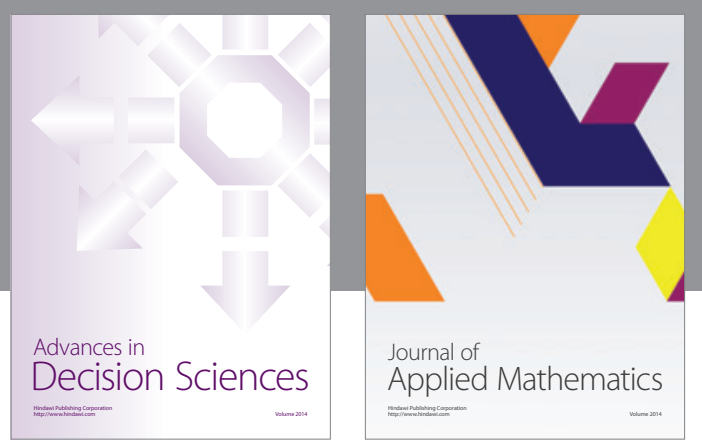

Algebra

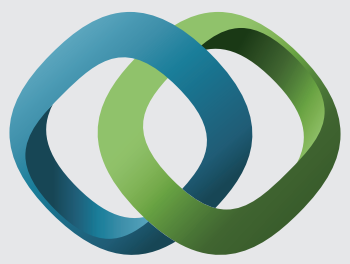

\section{Hindawi}

Submit your manuscripts at

http://www.hindawi.com
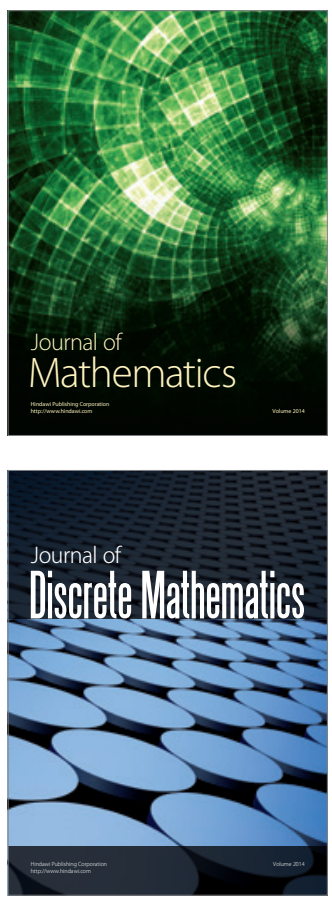

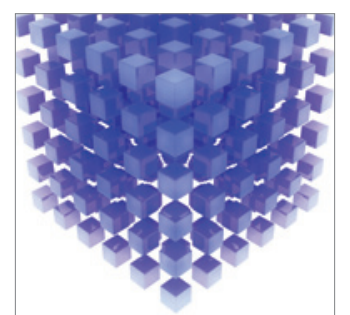

Mathematical Problems in Engineering
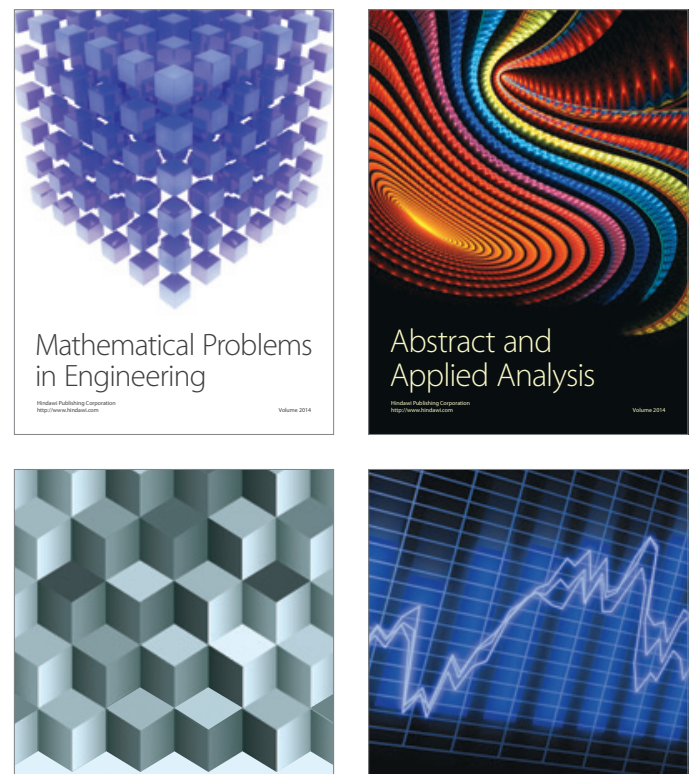

Journal of

Function Spaces

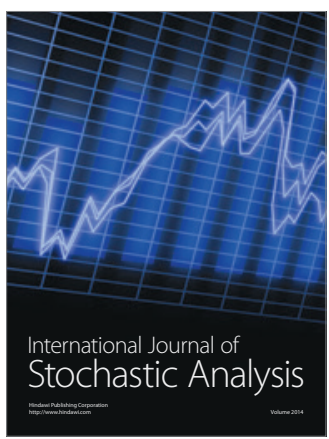

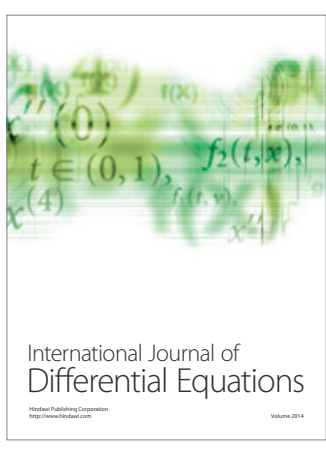
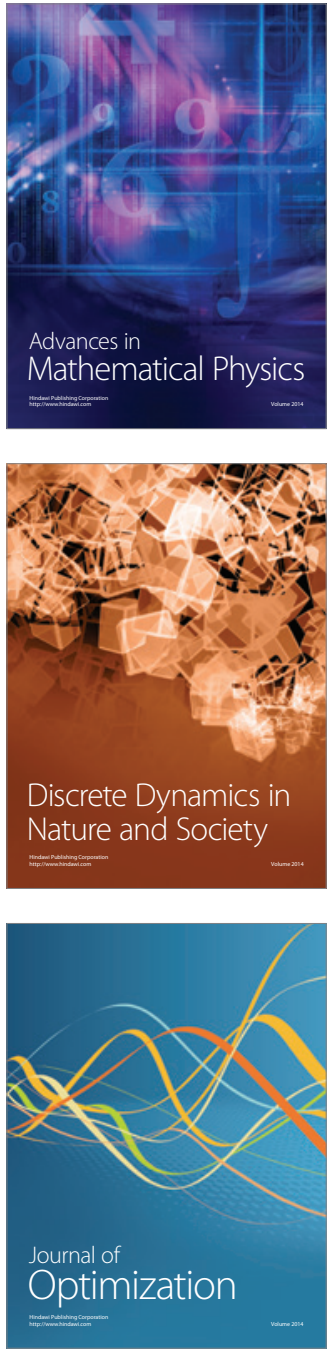